\title{
Research on Access Rate Optimization Algorithm in D2D Communication System
}

\author{
Yanliang $\mathrm{Ge}^{1}$, Qiannan Zhao ${ }^{1}$, Guanghua Zhang ${ }^{1, *}$, Weidang $\mathrm{Lu}^{2}$ \\ ${ }^{1}$ Northeast Petroleum University, Daqing 163318, China; \\ ${ }^{2}$ Zhejiang University of Technology, Hangzhou 310014, China; \\ *dqzgh@nepu.edu.cn
}

\begin{abstract}
In the uplink mode based on cellular network, device to device (D2D) multi-user shared channel can effectively improve the spectrum utilization and overall communication capacity of the system, but it will also bring serious interference problems, which will affect the end-user experience. To solve this problem, an access rate optimization algorithm based on interference minimum allocation multiplexing resources is proposed in this paper. In the communication system where D2D users and cellular users coexist, the objective function of minimum interference is established firstly, and then the Hungarian algorithm is used to optimize the objective function, so that the total interference of D2D users in the system is the minimum, and the maximum number of D2D users accessing the system is achieved. The simulation results show that the algorithm can reduce the interference of D2D users to cellular users in a certain range, and also increase the number of D2D users accessing the system to a certain extent.
\end{abstract}

Keywords: D2D communication, Cellular network, Resource allocation, Interference control.

\section{Introduction}

With the rapid development of wireless communication technology and the rapid popularization of intelligent terminal equipment, with the exponential growth of network traffic, it puts tremendous pressure on operators' core networks and spectrum, and mobile networks are facing severe challenges [1]. Device to device (D2D) communication technology is also called terminal pass-through technology. It directly implements data exchange and service provision without the need for base station transfer. The introduction of D2D communication in the cellular network can reduce the burden of the base station, reduce mobile terminal power consumption, improve transmission efficiency, and increase spectrum Utilization, improve system performance.

Under the control of the base station, D2D communication technology can use cell resources in orthogonal mode or multiplex cell resources in non-orthogonal mode (multiplexing mode) for communication, at present, most researches on D2D communication in multiplex mode [2]. In the multiplexing mode, the introduction of D2D 
communication technology into the cellular networks will bring serious interference problems [3]. How to allocate resources to reduce interference becomes the research focus of D2D communication.

In [4], an algorithm based on joint mode selection and resource allocation is proposed to achieve the maximum capacity and minimum interference of the communication system. In [5], a greedy heuristic algorithm is proposed, which takes the channel allocation problem as a mixed-integer nonlinear programming, and then improves the total throughput as much as possible under the requirement of ensuring the entire user's signal to interference ratio (SINR). In [6], according to the threshold of the signal-to-noise ratio of cellular users and D2D users, as well as the maximum transmitting power of user equipment, the transmitting power of D2D users is calculated, to ensure the quality of service of users. In [5,6], although the quality of communication service is guaranteed, the improvement of system throughput is limited. In [7], under the condition of ensuring the quality of service (QoS) cellular users and D2D users, the access set of D2D users is selected by linear programming to ensure that the interference of D2D communication will not affect cellular communication; secondly, a channel allocation scheme is proposed to maximize system throughput. In [8], a scheme of resource allocation and power control is proposed to ensure the interference of cellular link is controllable. In [9], a simplified bipartite graph is proposed, which uses the Hungarian algorithm for resource allocation to maximizing the rate. In [10], the authors propose a priority heuristic algorithm of D2D for multiplexing channel resources between users and cellular users, which can dynamically adjust the multiplexing channel resources, effectively increase the number of users allowed for D2D communication, and reduce the interference to cellular users. In [11], the resource allocation problem of D2D users is transformed into a parameter planning problem that can be solved by the Dinkelbach algorithm, and joint power control, relay selection and resource allocation scheme is obtained by the Hungarian algorithm. In [12], It guarantees the quality of service (QoS) requirements of users and puts forward a resource allocation method of grouping based on the greatly improved access rate. However, the system throughput of this scheme is relatively low, and once the number of D2D users increases, it cannot guarantee that the distance between D2D users in the group is within a reasonable range.

In the allocation of D2D multi-user shared channel, reduce the interference between cellular users and D2D users and maximize the number of D2D users accessing the system, to allocate communication resources reasonably. In the communication system where D2D users and cellular users coexist, a scheme is proposed to allocate multiplexing resources according to the minimum interference.

\section{System Model}

For the convenience of research, this paper only considers the scenario of D2D users multiplexing cellular communication resources in the uplink. Suppose the base station (BS) is in the center of the cellular system, there are also M cellular users (CU) in the system, and the set is expressed as $C=\left\{C U_{1}, C U_{2}, \cdots, C U_{m}, \cdots, C U_{M}\right\}$, K D2D 
users (DU), the set is expressed as $D=\left\{D U_{1}, D U_{2}, \cdots, D U_{k}, \cdots, D U_{K}\right\}$ and $\mathrm{N}$ uplink channel, CU and DU are randomly assigned in the system. DU exists in pairs, each D2D user pair includes D2D transmitting user (DUT) and D2D receiving user (DUR), In this paper, it is referred to as $\mathrm{T}$ and $\mathrm{R}$ for short. In Figure 1, the system model is shown.

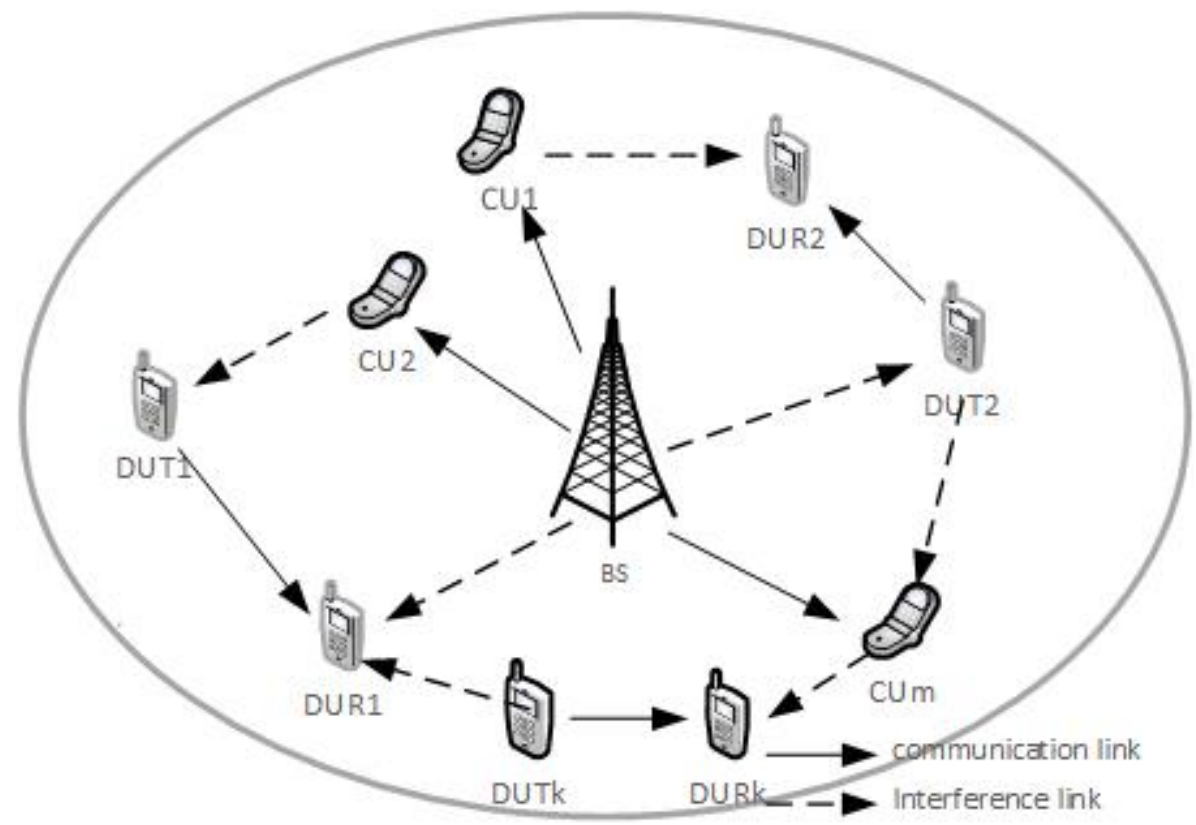

Fig. 1. System model

Compared with the downlink, the spectrum utilization of uplink communication links in the cellular networks is lower. and the interference of the receiver of D2D users is relatively less when multiplexing the uplink communication. At the same time, the base station can also control the spectrum resources in the system according to its interference. Therefore, this paper only considers the sharing scenario of uplink resources in a single cell. To ensure the communication quality, at most one DU can be reused for one $\mathrm{CU}$, and at most one CU can be reused for a pair of DU users.

\section{Problem Description}

When DU reuses the resources of CU, it will be interfered with by CU and other DU. In addition, DU will also interfere with the base station. In order to reduce the interference between cellular users and D2D users and allocate the communication resources reasonably, this paper establishes a system optimization model. In the system model of Figure 1, in order to improve the communication efficiency, when the kth 
D2D pair multiplexes the communication link of the mth cellular user, the receiver of the D2D user will be interfered by the uplink cellular channel, so the signal interference ratio of the $\mathrm{D} 2 \mathrm{D}$ receiver meets the following requirements:

$$
\operatorname{SINR}_{k, m}=\frac{G_{k} P_{k, m}}{N_{0}+A_{m, k}} \geq T_{0}
$$

Where $G_{k}$ is the channel gain between $R_{k}$ and $T_{k}$ of the D2D user, $P_{k, m}$ s the transmission power of $T_{k}$ multiplexing the mth uplink cellular user, $N_{0}$ is the white noise received by $R_{k}, A_{m, k}$ is the interference value of DU link caused by the interference of CU link. In order to ensure the normal and effective communication of DU, it is necessary to meet the requirement that the signal to interference ratio is at least $T_{0}$. Then transmit power $T_{k}$ needs to meet:

$$
P_{k, m} \geq P_{k, m}^{(0)}
$$

When $D U_{k}$ multiplexes $C U_{m}, T_{k}$ needs to achieve the minimum transmit power.

$$
P_{k, m}^{(0)}=\frac{T_{0}\left(N_{0}+A_{m, k}\right)}{G_{k}}
$$

According to the system model in Figure 1, it is known that the D2D user may also cause interference to the base station when multiplexing the cellular user, and the interference to the base station when multiplexing the mth cellular user channel for the kth D2D user:

$$
I_{k, m}=P_{k, m} H_{K}
$$

Where $H_{k}$ is the channel gain between $T_{k}$ and base station.

According to Shannon formula, the throughput of D2D users when multiplexing cellular channel as:

$$
C_{k, m}=\sum_{k=1}^{K} \sum_{m=1}^{M} \sum_{n=1}^{N} \log _{2}\left(1+\operatorname{SINR} R_{k, m}^{n}\right)
$$

Among them, $C_{k, m}$ is the throughput when multiplexing the mth channel for the kth D2D user, and $S I N R_{k, m}$ is the maximum signal to interference ratio obtained under multiplexing cellular user communication. 
In order to maximize the capacity of the system, the essence is to find the maximum SNIR. As formula (1) (4) shows, formula (5) of maximum capacity problem can be further transformed into the problem of minimum interference.

$$
\min \sum_{k=1}^{K} \sum_{m=1}^{M} \sum_{n=1}^{N} \beta_{k, m}^{n} I_{k, m}^{(n)}
$$

$$
\text { s.t }\left\{\begin{array}{l}
(6.1) \beta_{k, m}^{(n)}=\{0,1\}, \forall k, m, n \\
(6.2) \sum_{k=1}^{K+1} \sum_{m=1}^{M+1} \beta_{k, m}^{n}=1, \forall n \\
(6.3) \sum_{k=1}^{K+1} \sum_{n=1}^{N} \beta_{k, m}^{n}=1, \forall m \\
(6.4) \sum_{m=1}^{M+1} \sum_{n=1}^{N} \beta_{k, m}^{n}=1, \forall n
\end{array}\right.
$$

Where $\beta_{k, m}^{n}$ means that the kth pair of DU can reuse the nth channel resource shared by the $\mathrm{CU}$, and $1 \leq n \leq N, 1 \leq m \leq M+1,1 \leq k \leq K+1$. When allocating resources in the actual communication system, N, M, K need to meet $K<M<N<K+M$. Part of the DU is allocated as a dedicated channel resource, so the Du will not be interfered with by other users, and does not interfere with the CU. The other part of the DU needs to reuse CU resources, and reasonable resource allocation needs to be made to make the access system with as little interference as possible and as many D2D users as possible. If $n \leq N, m \leq M, k \leq K$, $\beta_{k, m}^{(n)}=1$ means that $\mathrm{DU}$ and $\mathrm{CU}$ may reuse channel $\mathrm{n}$; if $n \leq N, m=m+1, k \leq K, \beta_{k, m}^{(n)}=1$ means that the DU uses a dedicated channel $\mathrm{n}$; if $n \leq N, m \leq M, k=K+1, \beta_{k, m}^{(n)}=1$ means that the CU uses a dedicated channel $\mathrm{n}$; if $k=K+1, m=M+1, n \leq N, \beta_{k, m}^{(n)}=1$ means that dedicated channel $\mathrm{n}$ is idle.

\section{Resource Allocation Algorithm}

\subsection{Algorithm Analysis}

The algorithm proposed in this paper is to ensure the communication quality of the system and maximize the communication capacity of the system, and then carry out a reasonable allocation of user communication resources so that the performance of the communication system reaches the optimal and D2D users access the system most. 
The algorithm steps are as follows: First, construct an interference matrix, and again based on the combination of cellular users, D2D users, and channels in the matrix, and find the smallest interference, finally, resource allocation is based on this data. Because there are $\mathrm{m}$ free channels left in the system, when allocating the free channels to users, only specific cellular users need to be determined to be allocated to specific D2D users for multiplexing, while the free channels are allocated to the remaining D2D users. Suppose all communication channels are the same. In order to ensure the normal communication quality, the interference threshold value of the received information of the base station is preset as $I_{0}$.

(1) If $I_{k, m}^{(n)} \leq I_{0}$, means that when the kth pair of D2D users can reuse the nth channel resource of the mth cellular user and the interference value is less than the interference threshold received by the base station, access to the D2D pair of users is allowed. The access admission parameter is $\theta_{k, m}^{(n)}=1$; if $I_{k, m}^{(n)}>I_{0}$, means that the kth pair of D2D users cannot reuse the nth channel of the mth cellular user, then set the admission parameter as $\theta_{k, m}^{(n)}=0$.

(2) If D2D uses a dedicated channel $n$, it can be considered that the D2D user reuses the $n$ channel used by a cellular user with zero interference, so we assume $I_{k, m+1}^{(n)}=0$. If the dedicated channel is used by a cellular user, it is considered that the cellular user and a D2D user with zero interference to the base station multiplex the nth channel, so we assume $I_{m, k+1}^{(n)}=0$. In this way, we can calculate an admission matrix of $N \times(m+1) \times(k+1)$, which is $\theta_{k+1, m+1}^{(n)}$, and an interference matrix of $N \times(m+1) \times(k+1)$ is $I_{m+1, k+1}^{(n)}$.

(3) From equation (4), we can know that the problem of maximum capacity is further transformed into the problem of minimum interference, and then it is transformed into the 0 -1 assignment problem of the interference matrix for resource reuse. Therefore, in the channel resource reuse allocation, the obtained interference matrix is applied to the Hungarian algorithm to find the optimal resource reuse strategy ${ }^{[13]}$.

(4) In the dedicated channel allocation, it is only necessary to find the minimum interference value in the interference matrix $I_{K \times M}$, and then directly assign an uplink channel to the user for D2D.

\subsection{Simulation Parameters}

In the simulation, the communication cell is a circular cell with a radius of 500 meters. A base station exists in the center. D2D user pairs and cellular users are randomly and uniformly distributed in the cell. Each cellular user can only occupy one cellular channel, and each cellular channel is only allocated to one cellular user or one 
D2D pair user or one D2D pair user and one cellular user. Compared with the heuristic algorithm and the random algorithm, the algorithm proposed in this paper takes the interference threshold of the base station and the distance between the D2D transceiver users as variables, and its system allows the number of D2D users to access to increase significantly. Due to the random algorithm resource allocation, the D2D users in the system select the channel resource multiplexing randomly, without considering the interference between users, so the communication quality of D2D users cannot be guaranteed. But the algorithm improved in this paper is to establish the objective function of minimum interference, and use the Hungarian algorithm to achieve the optimization of this objective function, so that the D2D receiving end of the system is subject to the best pairing strategy with the least total interference, thus bringing the number of D2D users Increased access systems. By using MATLAB for simulation comparison, data is randomly generated each time and the average value is obtained. The main system simulation parameters are shown in Table 1.

Table 1. System simulation parameters.

\begin{tabular}{ll}
\hline Parameter & Value \\
\hline Cell radius/m & 500 \\
Total system bandwidth/MHz & 10 \\
Path loss model for D2D link/dB & $148+40 \lg d$ \\
Path loss model for cellular link/dB & $128.1+37.6 \lg d$ \\
D2D link shadow fading standard deviation/dB & 12 \\
Cellular link shadow fading standard deviation/dB & 10 \\
Noise spectral density/(dBm/Hz) & -174 \\
D2D receiver SINR threshold/dB & -10 \\
\hline
\end{tabular}

\subsection{Analysis of Simulation Results}

Figure 2 shows the relationship between the interference threshold of the base station and the logarithm of system access D2D under different algorithms. It can be seen from the figure that when the interference threshold of the base station is increasing, the D2D allowed by the system to access user communication is also increasing. When the interference threshold of the base station is about - 70dBm, the D2D logarithm of the three algorithms can reach the maximum allowable access value of the system. It can be seen from Figure 2 that the algorithm and the heuristic algorithm have a smaller difference between the number of D2D users allowed by the system when the interference threshold of the base station increases. So the system allows fewer D2D users to access. However, compared with the comparison algorithm, when the interference threshold of the base station is between - 150dbm to $120 \mathrm{dbm}$, the number of users allowed to access D2D increases faster with the increase of the threshold. 


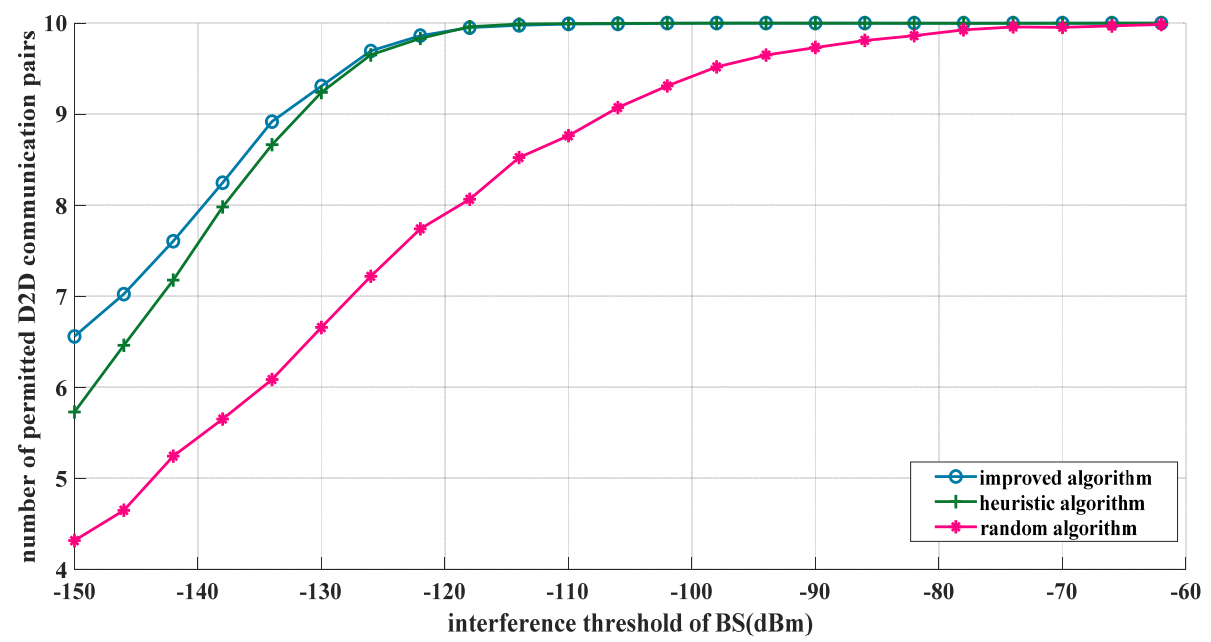

Fig. 2. The relationship between the interference threshold of the base station and the log of D2D

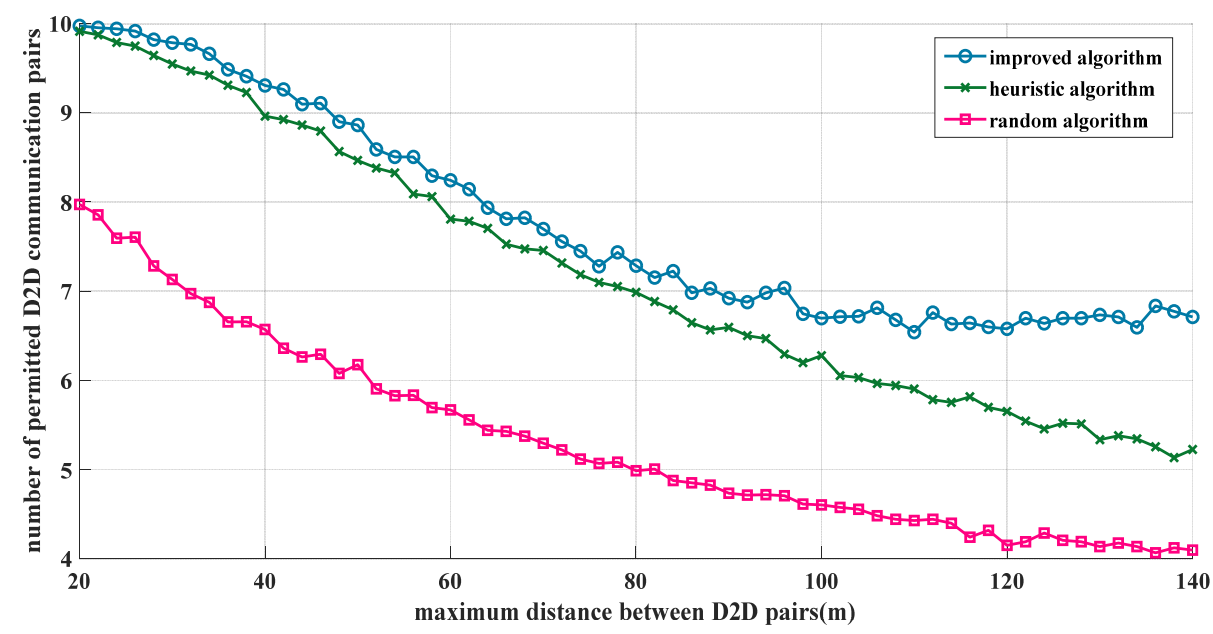

Fig. 3. The relationship between the distance between D2D users and the logarithm of users accessing D2D.

Figure 3 shows the maximum distance between the users of D2D receiving and sending end between $20 \mathrm{~m} \sim 140 \mathrm{~m}$ and the logarithm relationship between the users of D2D allowed to access the system. It can be seen that in the process of increasing the maximum distance, the D2D allowed to access keeps more access to the number of users. As can be seen from Figure 3, as the distance between the users of the D2D transceiver increases, the number of D2D users allowed to access the system gradually decreases, which is caused by the more interference the more D2D users. Moreover, the system of the algorithm in this paper allows the number of D2D users to ac- 
cess to decrease significantly less than heuristic and random algorithms. When the maximum distance between D2D transceiver users is $20 \mathrm{~m} \sim 100 \mathrm{~m}$, the algorithm and heuristic algorithm in this paper can maintain a higher number of D2D users accessing the system, but as the maximum distance between D2D transceiver users increases, the interference gradually increases. When the maximum distance between D2D transceiver users is between $100 \mathrm{~m}$ and $140 \mathrm{~m}$, the resource allocation algorithm based on the objective function of minimum interference in this paper makes the number of D2D users allowed to be accessed by the system significantly higher than its comparison algorithm.

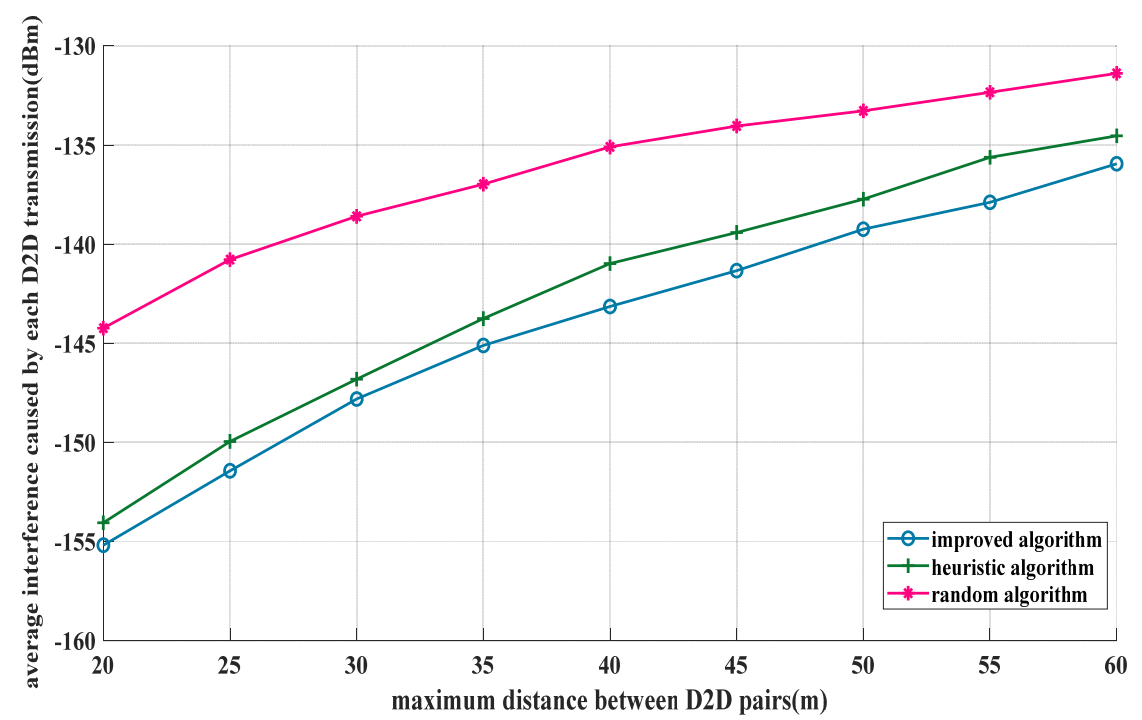

Fig. 4. The relationship between the distance between users of D2D and the average interference caused by each pair of D2D.

Figure 4 shows the relationship between the maximum distance between the users of D2D receiving and sending end and the average interference value caused by each pair of D2D users. The interference value of each pair of D2D users increases with the increase of the maximum distance of D2D receiver and transmitter users. With the increase of the distance between the receiver and the transmitter, the transmitting power of D2D to the user's transmitter is also increasing, so the interference will also increase. When the maximum distance between the transmitter and receiver of D2D is $30 \mathrm{~m} \sim 60 \mathrm{~m}$, the interference caused by the improved algorithm is obviously lower than other algorithms.

\section{Conclusion}

This paper studies the resource allocation problem of multiple D2D and multiple uplink cellular users in a single cellular system by establishing the communication mod- 
el of D2D communication technology introduced into the cellular network, and carries out theoretical design and simulation analysis, so as to obtain the resource allocation scheme with the minimum interference as the target. This model takes the interference threshold of the base station and the distance between D2D communication pairs as variables to analyze, and optimizes the resource allocation process of D2D communication. The simulation results show that under the condition of limited communication resources and guaranteed user service quality, the algorithm maximizes the number of users of D2D access to the maximum extent allowed by the system, and reduces the interference of D2D to users of cellular users within a certain range. However, this system model only studies the interference of a single cell and does not consider the interference of neighboring cells, so the resource allocation of the multi-cell system model can be considered on this basis.

\section{References}

1. Zhou Yiqing, Pan Zhengang, Zhai Guowei, et al.: Research on the standardization outlook and key technology of 5G mobile communication system. Journal of Data Acquisition and Processing 30(4), 714-724 (2015).

2. Wang Junyi, Gong zhishuai, Fu Jielin, et al.: Overview of D2D communication technology. Guilin University of electronic technology Journal of science (2), 114-119 (2014)

3. Wang H, Leung S H, Song R.: Uplink Area Spectral Efficiency Analysis for Multichannel Heterogeneous Cellular Networks With Interference Coordination. IEEE Access 6(1), 14485-14497 (2018).

4. Yu G, Xu L, Feng D, et al.: Joint mode selection and rescource allocation for device-todevice communications. IEEE Transactions on Communications 62(11), 3814-3824 (2014).

5. Zulhasnine M, Huang C, Srinivasan A.: Efficient resource allocation for device-to-device communication underlaying LTE network [C]// Proc of IEEE International Conference on Wireless and Mobile Computing, NETWORKING and Communications.2010:368-375.

6. Oduola W O, Li X, Qian L, et al. Power control for device-to-device communications as an underlay to cellular system [C]// Proc of IEEE International Conference on Communications. 2014:5257-5262.

7. Huynh T, Onuma T, Kuroda K, et al.: Joint downlink and uplink interference management for device to device communication underlaying cellular networks. IEEE Access 4, 44204430 (2016).

8. Wang Rong, Jiang Fan, Xu Tengju, et al.: A scheme of combining resource allocation and power control in D2Dcommunication. Telecommunication Technology (3), 295-301 (2016).

9. WANG L, TANG H, WU H Q, et al.: Resource allocation for D2D communications underlays in rayleigh fading channels. IEEE Transactions on Vehicular Technology 66(2), 1159-1169 (2017).

10. Xu Y, Yin R, Han T, et al.: Dynamic resource allocation for device-to-device communication underlaying cellular networks. International Journal of Communication Systems 27(10), 2408-2425 (2012).

11. Qu H, Zhu Z C, Zhao J H, et al.: Energy-Efficient joint relay selection and resource allocation scheme for mobile relay aided device-to-device communication. Journal of Electronics \&Information Technology 39(10), 2464- 2471 (2017). 
12. VAN S D, SHIN Y, SHIN O S.: Resource allocation and power control based on user grouping for underlay Device-to-Device communications in cellular networks. Transactions on Emerging Telecommunications Technologies 28(1), 1-12 (2017).

13. Li YuanZuo, Yang Xiaoduan.: Improved algorithm of assignment problem. Value engineering 34 (07), 303-304 (2015). 